\title{
Comportamento de compactação de mix argila/resíduo de pó de pedras
}

\section{(Compaction behaviour of clay/stones powder waste mix)}

\author{
M. N. Freire, S. J. G. Souza, A. G. P. Silva, J. N. F. Holanda \\ CCT-LAMAV, Universidade Estadual do Norte Fluminense \\ Campos dos Goytacazes, RJ, 28013-602 \\ holanda@uenf.br
}

\begin{abstract}
Resumo
Este trabalho apresenta os resultados de um estudo sobre o comportamento de compactação de massas cerâmicas contendo resíduo proveniente da indústria de pedras de Santo Antônio de Pádua-RJ. Para este propósito, cinco massas foram preparadas contendo até $20 \%$ em peso de resíduo. As características com respeito a difração de raios X, morfologia, distribuição de tamanho de partículas e massa específica real dos grãos foram determinadas. As massas foram submetidas a processo de compactação a frio com pressões de até $60 \mathrm{MPa}$, e avaliadas a partir dos diagramas de resposta de compactação e taxa de compactação. Para cada massa cerâmica foi determinada uma expressão matemática que descreve com precisão a relação entre a densidade e a pressão de compactação. Os resultados revelaram que a compactação das massas é governada, até a pressão aplicada de $60 \mathrm{MPa}$, por dois mecanismos de compactação dominantes incluindo o rearranjo de partículas e a deformação plástica. A taxa de densificação é inicialmente alta, e depois diminui rapidamente para pressões acima de 2,45-3,22 MPa. Além do mais, foi determinado que com o aumento do conteúdo de resíduo incorporado as massas apresentaram melhor eficiência de compactação para as condições estudadas.
\end{abstract}

Palavras-chave: resíduo de pó de pedras, compactação, reciclagem, massas cerâmicas.

\begin{abstract}
This work presents the results of a concerning study on the compaction behaviour of stones powder waste containing ceramic masses. The stones powder waste was generated by the industries from Santo Antonio de Pádua-RJ. For this purpose, five masses were prepared containing up to $20 \mathrm{wt} \%$ waste, and their characteristics regarding X-ray diffraction, particle size analysis, morphology, and grain real specific mass were determined. The masses were submitted the cold compaction process at pressures up to $60 \mathrm{MPa}$, and their compaction response and compaction rate diagrams were generated. For each ceramic mass was determined a mathematics expression that describe with precision the relationship between the density and compaction pressure. The results revealed that the compaction is governed, at the applied pressure range, by two dominant mechanisms including particle rearrangement and plastic deformation. The rate of densification is high initially, but then decreases rapidly for pressures above about $2.45-3.22 \mathrm{MPa}$. In addition, it was found that the incorporated masses with stone powder waste presented better compaction efficiency for the given testing conditions.
\end{abstract}

Keywords: stone powder waste, compaction, recycling, ceramic masses.

\section{INTRODUÇÃO}

O município de Santo Antônio de Pádua, no Noroeste do estado do Rio de Janeiro, possui cerca de 350 pedreiras e serrarias de beneficiamento de rochas com diferentes aspectos e propriedades físicas e químicas. Dentre os vários tipos de rochas beneficiadas destaca-se as rochas graníticas utilizadas principalmente na construção civil e pavimentação de ruas, avenidas e calçadas. No processo de corte e beneficiamento dessas pedras são geradas grandes quantidades de resíduo sólido em forma de pó não biodegradável. Este resíduo vem sendo descartado sem nenhum controle, o qual poderá resultar em sérios impactos ao meio ambiente. Assim, deve-se buscar alternativas viáveis para minimização e/ou reciclagem deste resíduo, resultando em ganhos de ordem econômica e ambiental.

Uma área que tem tornado-se promissora na reciclagem de resíduos industrias é a da cerâmica vermelha [1-4]. Isto decorre fundamentalmente da larga variabilidade da composição químico-mineralógica das matérias-primas utilizadas em cerâmica vermelha. De fato, é possível fabricar produtos de cerâmica vermelha com matérias-primas que apresentam distintas características oriundas de diversos lugares do mundo [5]. Os trabalhos sobre incorporação de resíduos em massas argilosas são voltados, sobretudo, para o estudo de propriedades tecnológicas após queima, visando alcançar os valores especificados nas normas técnicas. Entretanto, a compactação que é um processo complexo tem sido pouco estudada a despeito da sua importância técnico-científica.

A compactação de pós secos ou semi-secos por prensagem uniaxial em matriz rígida, constitui-se num dos métodos de conformação de pós mais utilizados na indústria cerâmica $[6,7]$. A compactação é uma etapa intermediária do processamento de materiais cerâmicos. O conhecimento da relação entre a massa específica verde e a pressão de 
compactação é de alto interesse tecnológico. A pressão aplicada à massa de pó influencia a porosidade e a resistência mecânica do compacto verde. Assim, o entendimento dos mecanismos de consolidação de pós durante a compactação, contribui fortemente para o controle das propriedades dos compactos, resultando na obtenção de produtos acabados cada vez melhores. Um método rápido e eficiente de inferir sobre os mecanismos de compactação de pós é o diagrama de resposta de compactação (densidade relativa versus logaritmo da pressão) [8-10].

No presente trabalho foi feito um estudo no sentido de descrever o comportamento de compactação de massas cerâmicas contendo resíduo de pó de pedras. Avaliou-se o comportamento de compactação por meio de diagramas de resposta de compactação e taxa de compactação, objetivando inferir os prováveis mecanismos de compactação dominantes que governam a compactação no intervalo de pressões utilizado. Além disso, foi estudado o efeito da adição do resíduo sobre a densificação dos corpos cerâmicos durante o processo de compactação.

\section{MATERIAIS E MÉTODOS}

No presente trabalho foram preparadas cinco massas cerâmicas contendo adições de até $20 \%$ em peso de resíduo de pó de pedras (Tabela I). No preparo dessas massas, foi usada uma massa argilosa industrial, predominantemente caulinítica [11], fornecida por uma empresa cerâmica instalada no município de Campos dos Goytacazes-RJ. O resíduo, gerado no processo de desmonte de pedras, foi fornecido por uma empresa instalada no município de Santo Antônio de Pádua-RJ.

A preparação das massas consistiu da secagem das matériasprimas em estufa a $110{ }^{\circ} \mathrm{C}$ por um período de $24 \mathrm{~h}$, seguido de destorramento em pilão manual e peneiramento (-100 mesh). Em seguida foi feita a dosagem por pesagem e subsequente processo de mistura/homogeneização num misturador cilíndrico, por um período de $1 \mathrm{~h}$. As massas devidamente secas foram umedecidas a cerca de $7 \%$ (base seca), e armazenadas em sacos plásticos fechados num dessecador por $24 \mathrm{~h}$.

A análise de distribuição de tamanho de partículas das massas preparadas foi feita utilizando-se uma combinação de técnicas de peneiramento e sedimentação, de acordo com a norma NBR 7181-84 [12]. A massa específica real dos grãos foi determinada de acordo com a norma NBR 7180-84 [13]. A análise mineralógica por difração de raios $\mathrm{X}$ foi feita num difratômetro modelo URD 65, Seifert, utilizando-se radiação

Tabela I - Composição das massas cerâmicas (\% em peso). [Table I - Compositions of the ceramic masses (wt.\%).].

\begin{tabular}{ccc}
\hline Amostras & Argila & Resíduo \\
\hline M1 & 100 & 0 \\
\hline M2 & 95 & 5 \\
\hline M3 & 90 & 10 \\
\hline M4 & 85 & 15 \\
\hline M5 & 180 & 20 \\
\hline
\end{tabular}

$\mathrm{Cu} \mathrm{K}$. A análise qualitativa das fases presentes foi efetuada através da comparação do ângulo de Bragg e da distância interplanar dos principais picos experimentais obtidos, com fichas padrão JCPDS [14]. A morfologia das partículas das matérias-primas utilizadas foi determinada com o auxílio de um microscópio eletrônico de varredura modelo DSM 962, Zeiss.

As massas cerâmicas foram submetidas a compactação por prensagem uniaxial com ação única do pistão superior. As medidas de carga aplicada à massa de pó em função do deslocamento do pistão foram realizadas numa máquina universal de ensaios modelo 5582, Instron, capacidade de 100 $\mathrm{kN}$, com taxa de carregamento de $0,5 \mathrm{~mm} \cdot \mathrm{min}^{-1}$, e sistema para aquisição dos dados experimentais. As massas foram compactadas numa matriz cilíndrica de aço inoxidável de diâmetro interno de aproximadamente $15,85 \mathrm{~mm}$. A pressão de compactação máxima aplicada foi de $60 \mathrm{MPa}$.

Monitorando-se o deslocamento do cursor da máquina universal de ensaios durante a compactação é possível obter parâmetros importantes do compacto verde [15]. Tais parâmetros fornecem informações que permitem a construção de diagramas de resposta de compactação. Para melhor entendimento dos mecanismos de compactação foram construídos diagramas de taxa de compactação, $\mathrm{dDr} / \mathrm{dP}=\mathrm{f}(\mathrm{P})$, para cada massa preparada.

As amostras compactadas no intervalo de pressões utilizado foram fraturadas para acompanhamento por MEV das mudanças microtexturais e morfológicas durante a compactação.

\section{RESULTADOS E DISCUSSÃO}

A Fig. 1 mostra o difratograma de raios $X$ para a massa cerâmica contendo $20 \%$ em peso de resíduo de pedra (amostra M5). Nesta figura observa-se os picos característicos dos argilominerais e materiais não plásticos presentes na amostra. $\mathrm{O}$ argilomineral predominante é a caulinita $\left(\mathrm{Al}_{2} \mathrm{O}_{3} \cdot 2 \mathrm{SiO}_{2} \cdot 2 \mathrm{H}_{2} \mathrm{O}\right)$, sendo os seus picos apresentados de forma bem definida. Além da caulinita, é possível detectar picos característicos de argilomineral do grupo da mica, quartzo $\left(\mathrm{SiO}_{2}\right)$, gibsita $\left(\mathrm{Al}(\mathrm{OH})_{3}\right)$, microclina $\left(\mathrm{KAlSi}_{3} \mathrm{O}_{8}\right)$ e albita $\left(\mathrm{NaAlSi}_{3} \mathrm{O}_{8}\right)$.

A Fig. 2 mostra aspectos morfológicos do material argiloso e resíduo de pedras utilizados. Na Fig. 2a observa-se placas de material argiloso, provavelmente de caulinita. É possível observar também que algumas placas estão empilhadas formando aglomerados com diferentes tamanhos. Na Fig. 2 b nota-se que o resíduo de pedras apresentou partículas com morfologia tipicamente irregular com uma topografia superficial suave.

A distribuição de tamanho de partículas e as massas específicas real das massas cerâmicas são apresentadas na Tabela II. Os resultados mostram que todas as massas preparadas apresentam uma ampla distribuição de tamanho de partícula que varia desde $<2 \mu \mathrm{m}$ até $200 \mu \mathrm{m}$. A fração argila $(<2 \mu \mathrm{m})$, que está fundamentalmente relacionada com a presença de argilominerais, variou de $34 \%$ a $38 \%$. A fração silte $(2 \leq \mathrm{d}<60 \mu \mathrm{m})$ variou de $45 \%$ a $56 \%$. Enquanto que a fração areia $(>60 \mu \mathrm{m})$ variou de $7 \%$ a $19 \%$. Isto mostra claramente que as adições de resíduo alteraram a composição 


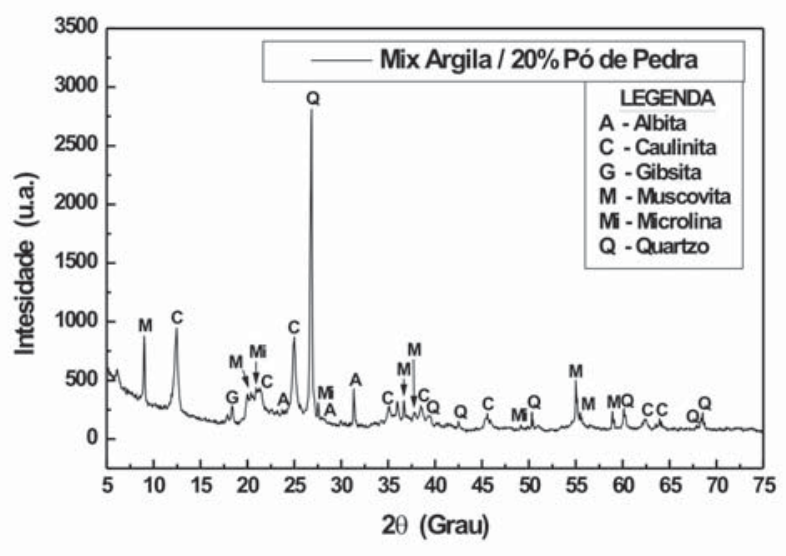

Figura 1: Difratograma de raios X da massa cerâmica M5. [Figure 1: X-ray diffractogram of the ceramic mass M5.].

a)

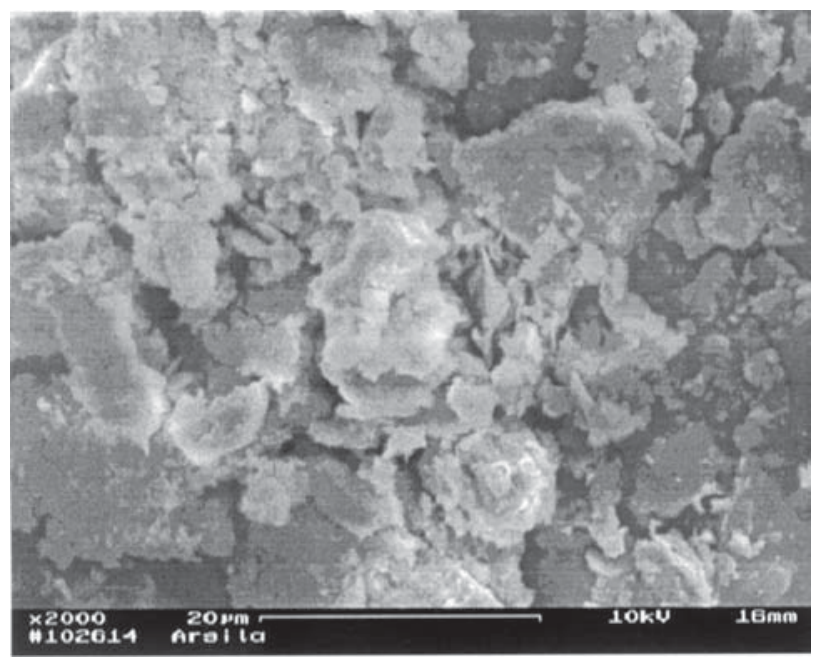

b)

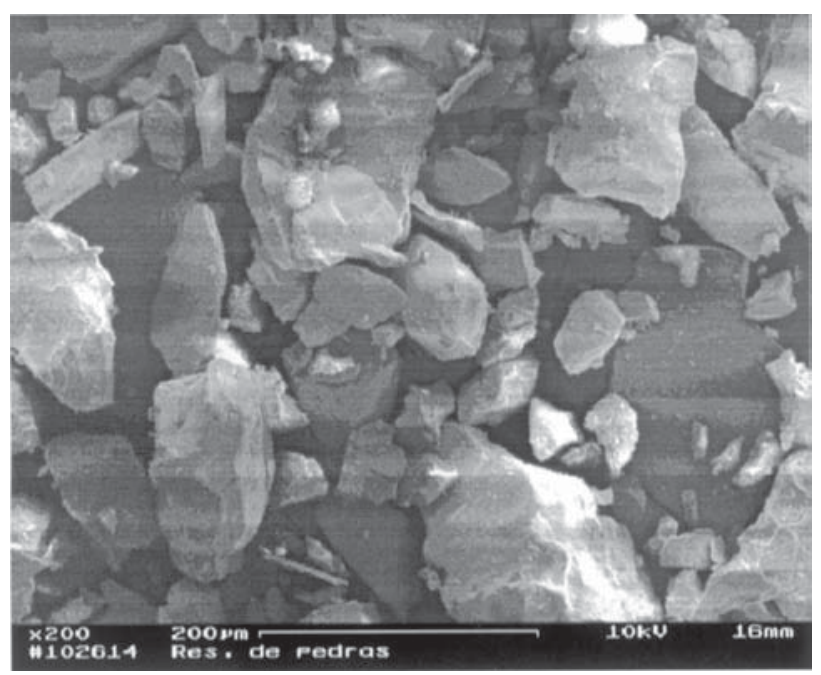

Figura 2: Aspectos morfológicos das partículas de pós: a) argila; b) resíduo.

[Figure 2: Overview of the powder particles: a) clay; b) waste sample.]. granulométrica das massas. À medida que se adiciona o resíduo aumenta-se o percentual de partículas com tamanho acima de $60 \mathrm{~mm}$. Nota-se, também, que há uma tendência de aumento da massa específica real dos grãos com o aumento do conteúdo de resíduo de pó de pedras.

O comportamento da curva da força aplicada em função do deslocamento do pistão é mostrado na Fig. 3, para todas as massas cerâmicas. Nota-se que, no intervalo de intensidade de força aplicada (valor máximo de $11.839 \mathrm{~N}$ ), às curvas estão associadas a diferentes energias $(9,39 \mathrm{~J}-10,30 \mathrm{~J})$ fornecidas pelo sistema (máquina universal de ensaios), para que seja mantida constante a velocidade do cursor da máquina. $\mathrm{O}$ comprimento da curva diminuiu com o aumento do conteúdo de resíduo de pedras. Assim, o sistema consumiu menos energia média (produto da intensidade da força média pelo módulo do deslocamento), no sentido de atingir a mesma pressão máxima desejada para todas as massas, que foi de $60 \mathrm{MPa}$.

Conhecendo-se a geometria da matriz e as dimensões finais dos compactos, foi possível determinar as massas específica verde e a pressão de compactação a partir da curva experimental força aplicada versus deslocamento do pistão (Fig. 3). Uma vantagem do método de compactação empregado é que obtém-se a curva de massa específica em todo o intervalo

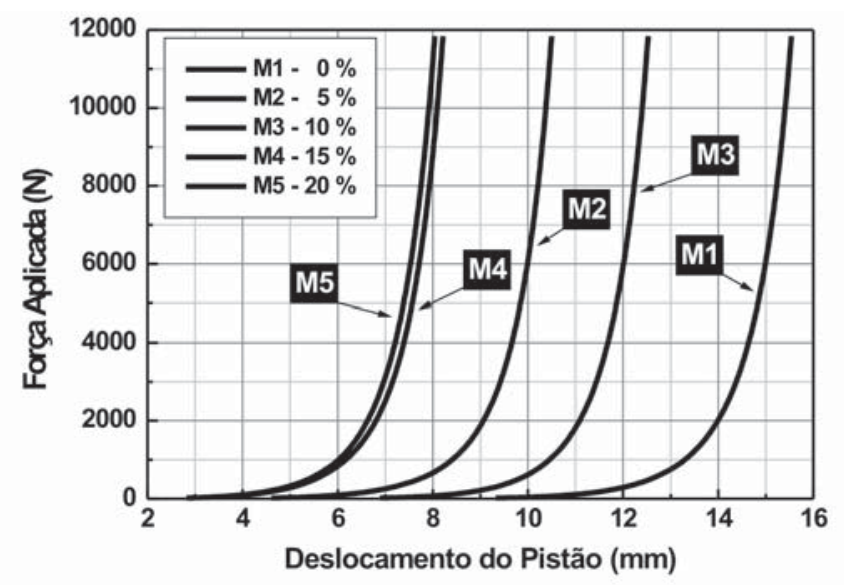

Figura 3: Curvas da força aplicada em função do deslocamento do pistão.

[Figure 3: Curves of applied force as a function of the plunger displacement.].

de pressões, somente com uma única amostra para cada massa cerâmica. As curvas de compactabilidade para todas as massas estudadas são mostradas na Fig. 4. Todas as curvas apresentaram um comportamento similar com elevada inclinação acima de determinado valor de pressão aplicada. Este comportamento pode ser explicado supondo que mecanismos de compactação distintos atuam em sequiência [16]. Nota-se claramente que o conteúdo de resíduo adicionado influenciou a compactação das massas. Os resultados indicam que, em geral, as massas contendo maior quantidade de resíduo apresentam compactabilidade superior. De fato para a massa M5, a massa específica verde máxima alcançada foi de $1,91 \mathrm{~g} / \mathrm{cm}^{3}$, obtida sob pressão de $60 \mathrm{MPa}$. A razão para isso deve-se ao fato que esta massa corresponde aquela com maior percentual de 


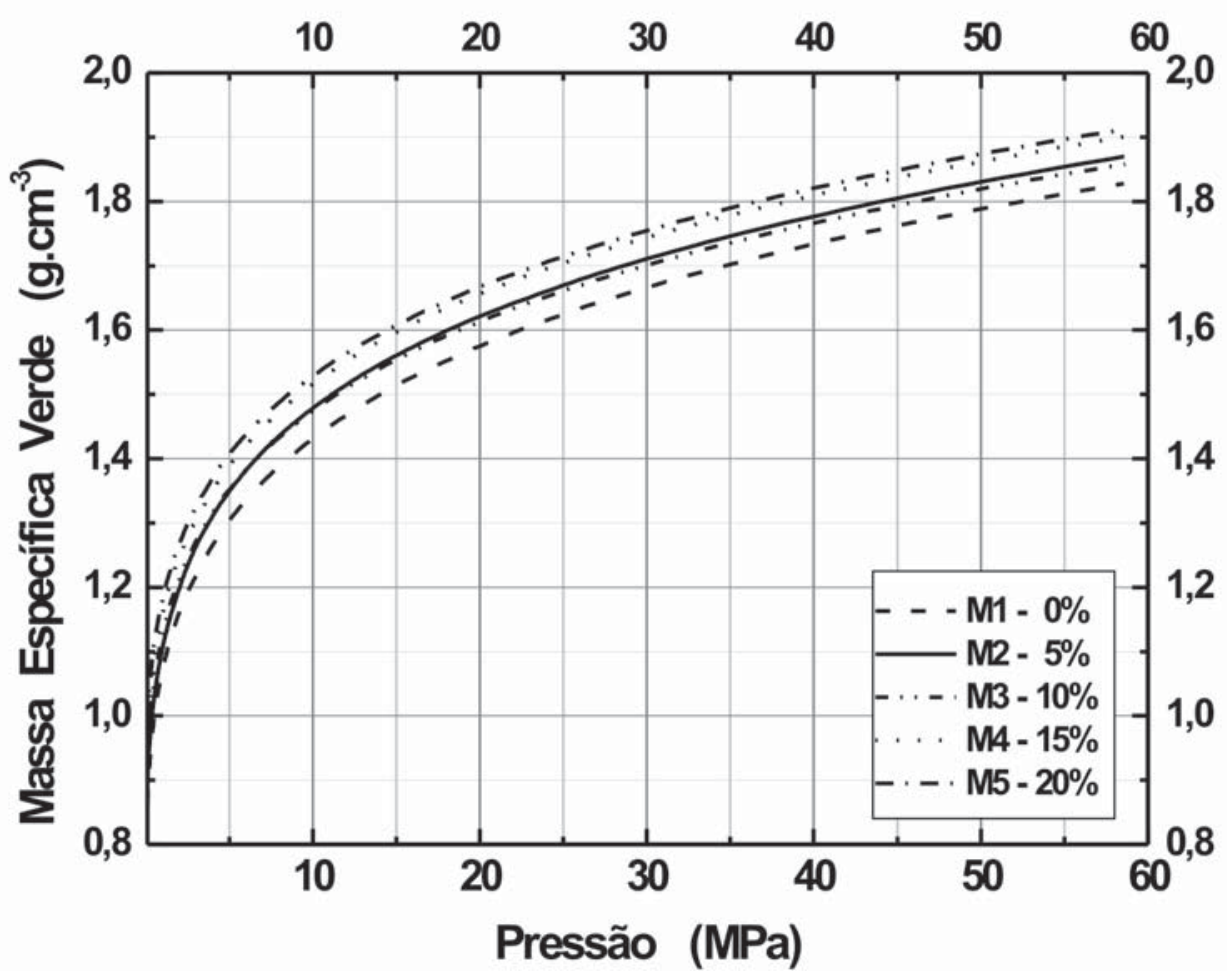

Figura 4: Massa específica verde em função da pressão de compactação. [Figure 4: Green density as a function of the compacting pressure.].

Tabela II - Distribuição de tamanho de partículas e massa específica real das massas preparadas. [Table II - Particle size distribution and specific mass for the prepared masses.]

\begin{tabular}{ccccc}
\hline Amostras & $\begin{array}{c}\text { Argila }(\%) \\
(<2 \mu \mathrm{m})\end{array}$ & $\begin{array}{c}\text { Silte }(\%) \\
(2 \leq \mathrm{d} \leq 60 \mu \mathrm{m})\end{array}$ & $\begin{array}{c}\text { Areia }(\%) \\
(60 \leq \mathrm{d} \leq 200 \mu \mathrm{m})\end{array}$ & $\begin{array}{c}\rho \\
\left(\mathrm{g}^{\circ} \mathrm{cm}^{-3}\right)\end{array}$ \\
\hline M1 & 37 & 56 & 7 & 2,68 \\
\hline M2 & 35 & 55 & 10 & 2,74 \\
\hline M3 & 38 & 47 & 15 & 2,70 \\
\hline M4 & 37 & 46 & 17 & 2,74 \\
\hline M5 & 34 & 47 & 19 & 2,75 \\
\hline
\end{tabular}

$\rho=$ massa específica real

Tabela III - Função polinomial e seus respectivos coeficientes numéricos para as massas cerâmicas. [Table III - Polynomial function and numerical coefficients of the ceramic masses.].

\begin{tabular}{cccccc}
\hline \multicolumn{5}{c}{$\mathrm{Dr}=\mathrm{a}+\mathrm{b} \log \mathrm{P}+\mathrm{c} \log ^{2} \mathrm{P}$} \\
\hline Amostras & $\mathrm{a}$ & $\mathrm{b}$ & $\mathrm{c}$ & $\mathrm{R}^{2}$ & $\mathrm{~s}$ \\
\hline M1 & 40,05081 & 10,49412 & 3,11438 & 0,99989 & 0,10585 \\
\hline M2 & 40,36678 & 10,57268 & 2,95934 & 0,99996 & 0,06030 \\
\hline M3 & 41,47631 & 10,14580 & 2,87125 & 0,99991 & 0,09112 \\
\hline M4 & 42,31327 & 9,956520 & 3,02586 & 0,99992 & 0,08885 \\
\hline M5 & 42,91803 & 9,68954 & 3,00869 & 0,99995 & 0,06441 \\
\hline
\end{tabular}

$D r=$ densidade relativa; $R^{2}=$ coeficiente de correlação; $s=$ desvio padrão 

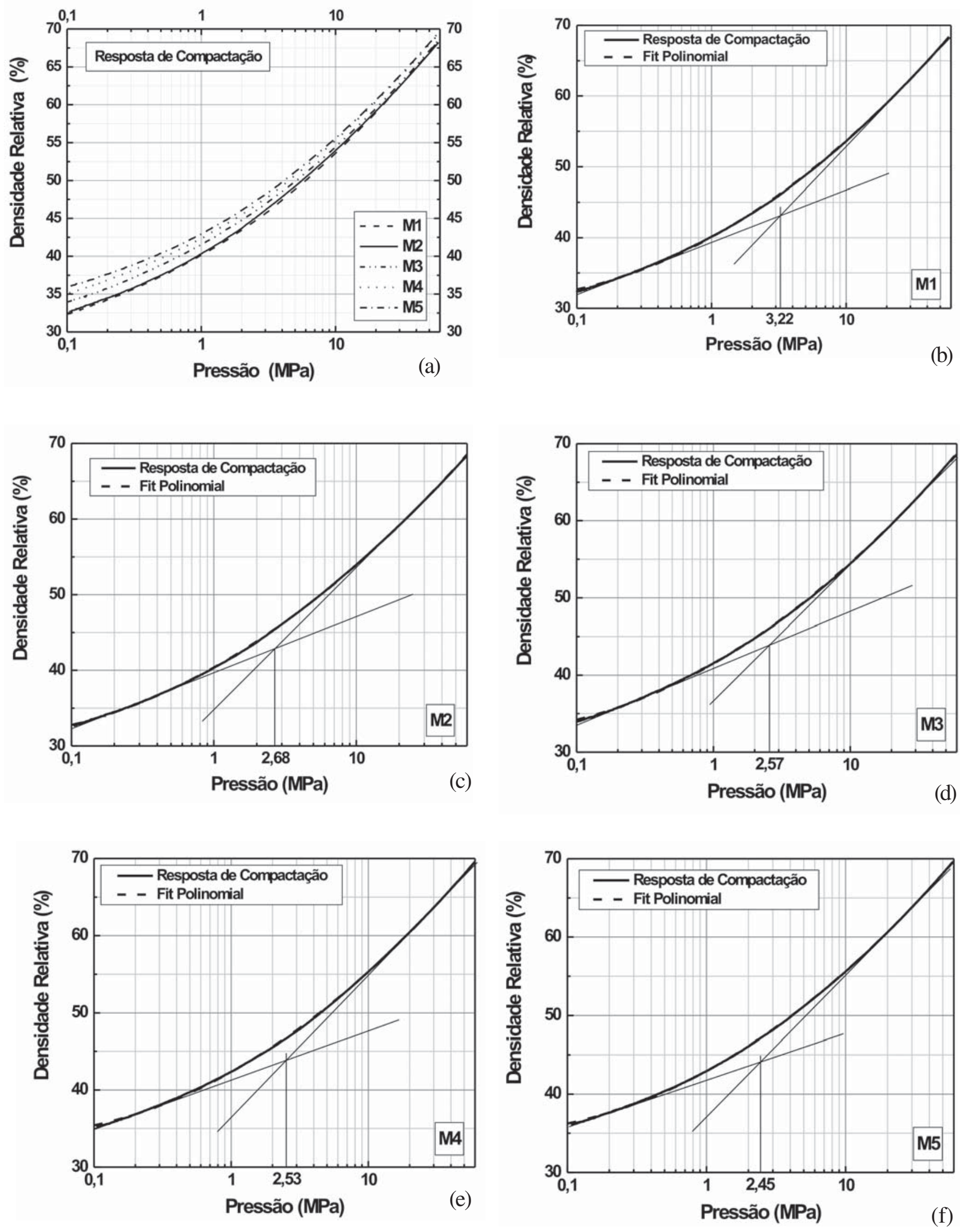

Figura 5: Diagramas de resposta de compactação para as massas cerâmicas. [Figure 5: Compaction response diagram for all ceramic masses.]. 

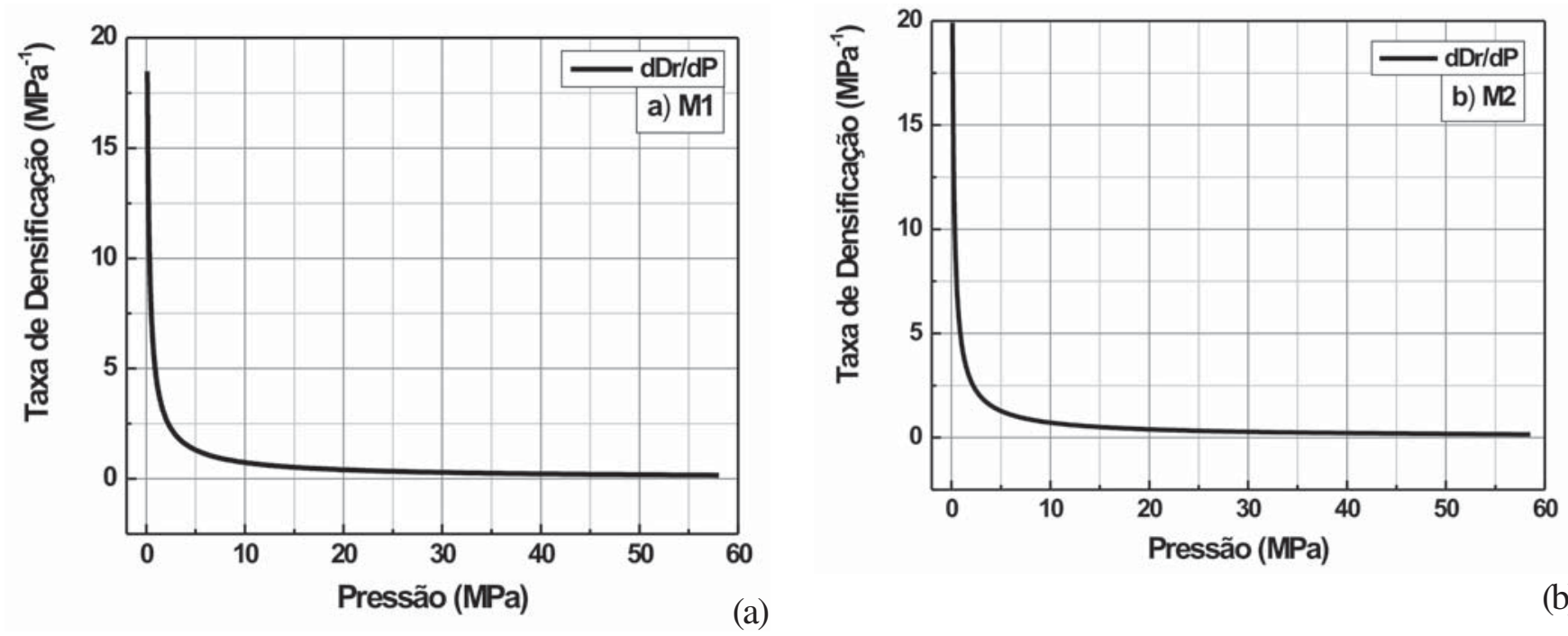

(a)

(b)
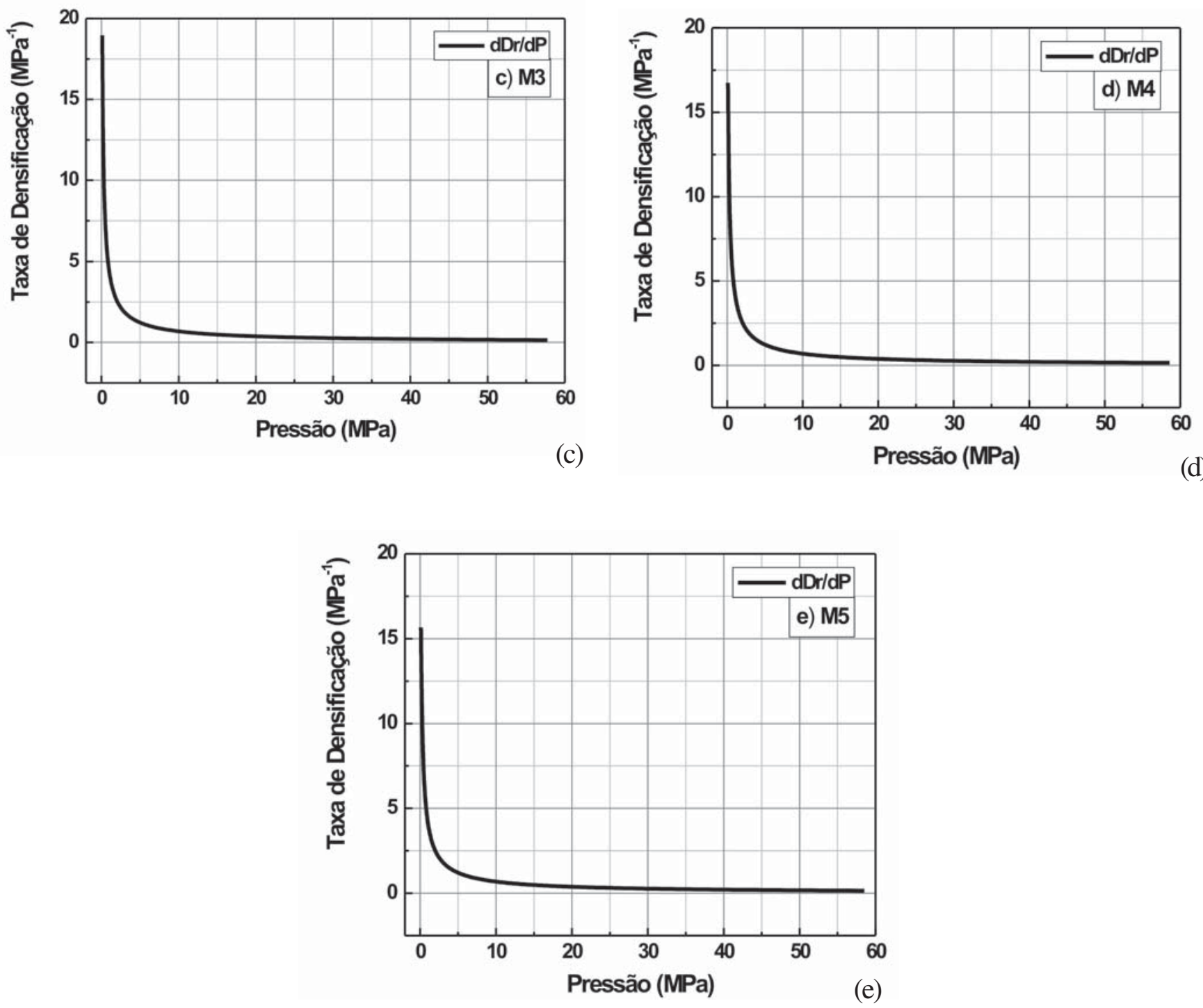

Figura 6: Diagramas de taxa de compactação para as massas cerâmicas.

[Figure 6: Compaction rate diagram for all ceramic masses.]. 
partículas acima de $60 \mathrm{~mm}$ (Tabela II). De modo que, as massas constituídas de partículas mais grossas sofreram um menor efeito do atrito entre as partículas e a parede da matriz. Como uma conseqüência, a força aplicada foi melhor transmitida resultando num empacotamento mais eficiente.

Os diagramas de resposta de compactação para todas as massas são mostrados na Fig. 5. A densidade relativa do compacto foi determinada usando os valores de massa específica real dos grãos dados na Tabela II. As curvas apresentaram um formato similar (Fig. 5a). No entanto, devem ser consideradas as diferenças nas curvas de resposta de compactação com relação a dois aspectos: i) a maior densidade relativa total no intervalo de pressão aplicado; e ii) a posição dos pontos de inflexão das curvas (Figs. 5b-5f). Nota-se que do ponto de vista de aumento da densidade relativa, a densificação do compacto aumentou à medida que se adiciona resíduo, em todo o intervalo de pressões. Isto mostra claramente que para as condições estudadas, a incorporação de resíduo de pedras teve um papel importante na densificação das massas cerâmicas. No entanto, tal comportamento observado não pode ser explicado somente com base nas diferentes granulometrias das massas. De acordo com a literatura [17], o tamanho, a geometria e a morfologia das partículas também devem ser considerados. As pressões nos pontos de inflexão $(\mathrm{Pe}=2,45$ 3,22 MPa) observados na Fig. 5 correspondem ao valor da pressão de escoamento aparente $(\mathrm{Pe})$ da massa de pó, na qual ocorre mudança de inclinação na curva de resposta de compactação. Isto pode ser interpretado como uma indicação da mudança do mecanismo que governa a compactação naquele intervalo de pressões. Os resultados mostram que há uma tendência de diminuição dos valores da pressão de escoamento com a adição de resíduo. A diminuição no valor de Pe implicou num aumento da compactabilidade das massas, conforme observado na Fig. 5.

Os resultados obtidos foram tratados matematicamente para expressar na forma analítica a relação entre a pressão de compactação e a densidade durante o processo de compactação. Os ajustes polinomiais mostrados nas Figs. 5b-5f, estão todos de forma coerentemente coincidindo com as curvas experimentais. As expressões matemáticas que regem estes ajustes são funções polinomiais quadráticas, que têm como incógnitas fatores essencialmente logarítmicos da pressão, e têm a mesma forma analítica para todas as massas, do tipo $\mathrm{y}=\mathrm{a}+\mathrm{bx}+\mathrm{cx}^{2}$, conforme mostrado na Tabela III. Nesta tabela são mostrados também os coeficientes de correlação e desvios padrões típicos, a partir dos quais pode-se verificar a validade das expressões matemáticas.

O diagrama da taxa de compactação, que corresponde a derivada primeira da curva de densidade relativa ( $\mathrm{dDr} / \mathrm{dP})$, é uma ferramenta útil para o entendimento dos processos que ocorrem durante a compactação $[10,17]$. Este diagrama mostra a taxa em que a compactação ocorre numa dada pressão. Os diagramas de taxa de compactação para as massas estudadas são mostrados na Fig. 6. O comportamento das curvas de taxa de compactação mostram claramente duas regiões distintas de compactação, corroborando com os resultados observados na Fig. 5. Estas regiões estão relacionadas a estágios distintos de compactação. A primeira região, equivalente ao intervalo de pressão abaixo do ponto de inflexão $(\mathrm{Pe})$, é caracterizada por uma maior taxa de aumento de densidade $(\mathrm{dDr} / \mathrm{dP})$. Nesta região o mecanismo

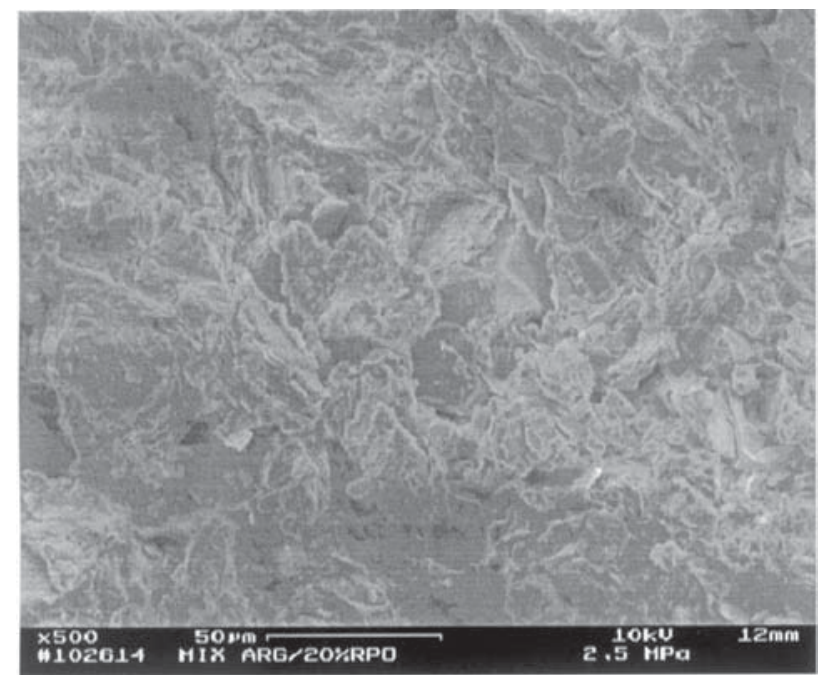

(a)

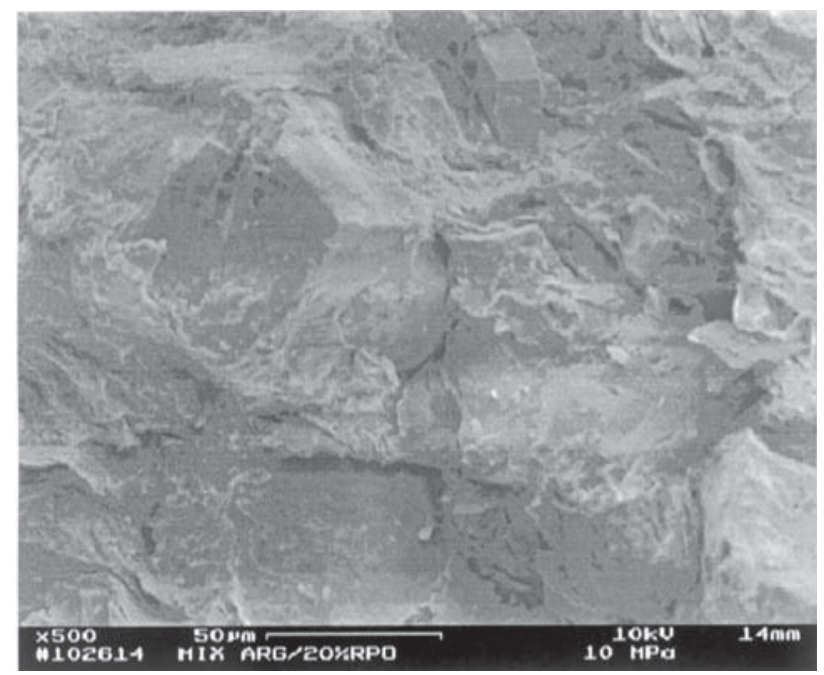

(b)

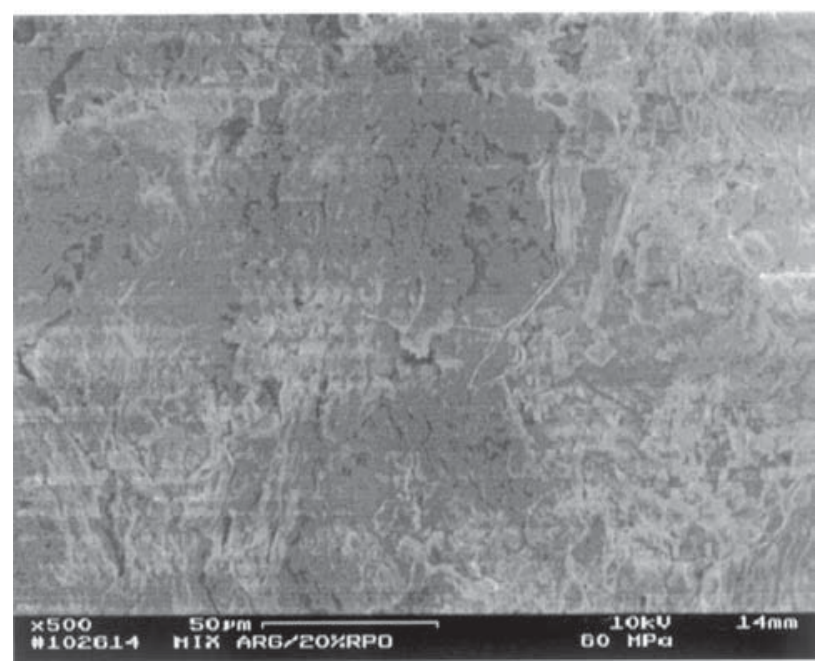

(c)

Figura 7: Microestrutura da amostra M5 compactada: a) 2,5 $\mathrm{MPa}$, b) $10 \mathrm{MPa}$, c) $60 \mathrm{MPa}$.

[Figure 7: Microstructure of the compacted sample M5: a) 2.5 $\mathrm{MPa}$, b) $10 \mathrm{MPa}, \mathrm{c}) 60 \mathrm{MPa}$.]. 
predominante que governa a compactação é o deslizamento e rearranjo das partículas. De fato, ocorreu um rearranjo das partículas, quando estas estavam sujeitas ao seu próprio peso e ao peso do pistão, e aplicou-se uma pressão relativamente pequena, causando mudanças no valor da densidade. Apesar da taxa de compactação ser o de maior valor em todo o processo, nessa região só se incrementa ligeiramente a compactação do corpo cerâmico. A segunda região, equivalente ao intervalo de pressão acima do ponto de inflexão (Pe), é caracterizado por uma menor taxa de aumento de densidade. No entanto, é nessa região que ocorrem grandes mudanças no valor da densidade. Tais mudanças ocorrem quando uma estrutura aberta está sendo fechada [17]. Amedida que a pressão é aplicada ocorre a remoção da porosidade intergranular com uma correspondente melhor configuração de empacotamento com uma estrutura mais homogênea, resultando num aumento da densidade do compacto. $\mathrm{O}$ mecanismo de deformação plástica domina o processo de compactação nesta região $[8,9]$.

A microestrutura de corpos prensados sob diferentes pressões (amostra M5) é mostrada na Fig. 7. Nota-se que quando a pressão de compactação aumentou de 2,5 MPa até $60 \mathrm{MPa}$, ocorreu uma grande diminuição da porosidade. Na Fig. 7 é possível também observar a transição de uma estrutura com alto grau de porosidade interconectada para uma com grande quantidade de poros isolados. Quando prensada sob 2,5 MPa (Fig. 7a), nota-se claramente um pobre empacotamento de partículas resultando em alta porosidade intergranular. Este valor de pressão é da mesma ordem da pressão de escoamento aparente da massa M5 $(\mathrm{Pe}=2,45 \mathrm{MPa})$, onde o mecanismo de densificação predominante é o rearranjo das partículas. Em $10 \mathrm{MPa}$ (Fig. 7b) o corpo cerâmico já apresenta uma melhor eficiência de empacotamento. Quando prensada sob $60 \mathrm{MPa}$ (Fig. 7c), nota-se que a maioria das partículas foram deformadas plasticamente, bem como ocorreu grande eliminação da porosidade intergranular. Além disso, pode-se notar que existe uma forte tendência para achatamento dos poros. Isto deve-se fundamentalmente a maior deformação na direção paralela à direção de prensagem, quando utiliza-se compactação em matriz rígida convencional.

Do ponto de vista industrial, a segunda região de compactação é a mais importante durante o processo [6]. Pois, esta região corresponde ao intervalo de pressões utilizado industrialmente, no sentido de atingir a massa específica verde desejada. O uso de pressões nesta região geralmente tem o efeito benéfico ao diminuir o parâmetro de retração linear e propiciar melhor vitrificação, resultando num aumento da resistência mecânica do corpo cerâmico acabado. De fato, o emprego de uma pressão adequada resulta invariavelmente num melhor contato entre as partículas, propiciando que todas as reações sejam aceleradas durante o processo de queima, inclusive a vitrificação [18].

\section{CONCLUSÕES}

O comportamento de compactação de massas cerâmicas contendo resíduo de pó de pedras foi investigado sob compactação a frio, utilizando-se diagramas de resposta de compactação e da taxa de compactação. Os resultados mostraram que a adição de resíduo melhorou a compactabilidade das massas, para as condições estudadas. Isto ocorreu devido a alterações nas composições granulométrica e mineralógica das massas causada pela incorporação de partículas do resíduo, principalmente acima de $60 \mu \mathrm{m}$. Foram observadas duas regiões de compactação distintas dependendo do intervalo de pressões empregado. Para a região que compreende desde pressões muito baixas até atingirse a pressão de escoamento $(\mathrm{Pe}=2,45-3,22 \mathrm{MPa})$, tem-se que o mecanismo de compactação dominante é o rearranjo de partículas com pouca influência na densidade dos corpos cerâmicos. Entretanto, foi nessa região que ocorreu a maior taxa de compactação. Para pressões acima da pressão de escoamento, que correspondeu a uma menor taxa de compactação, a compactação é governada por mecanismo de deformação plástica. Nesta região ocorreu grande redução da porosidade intergranular com concomitante aumento da densidade dos corpos cerâmicos. Além disso, o intervalo de pressões nesta região é o mais apropriado para fabricação de corpos cerâmicos.

\section{AGRADECIMENTOS}

Os autores agradecem a Cerâmica São José pelo fornecimento da argila e ao CNPq (Processo 551478/2002-0) pelo apoio financeiro.

\section{REFERÊNCIAS}

[1] S. A. Silva, Dissertação de Mestrado, Engenharia Ambiental, UFES-DEA, Vitória, ES (1998).

[2] G. P. Souza, Dissertação de Mestrado, UENF-PPGECM, Campos dos Goytacazes, RJ (2001).

[3] R. R. Menezes, H. S. Ferreira, G. A. Neves, H. C. Ferreira, Cerâmica 48, 306 (2002) 92-101.

[4] J. M. S. Moreira, Monografia, UENF-LAMAV, Campos dos Goytacazes, RJ, (2003) 40p.

[5] P. S. Santos, Ciência e Tecnologia de Argilas, Vol.1, 2 ${ }^{\mathrm{a}}$ Ed., Edgard Blücher Ltda., S. Paulo (1989).

[6] J. S. Reed, Principles of Ceramics Processing, $2^{\text {nd }}$ Edition, John Wiley \& Sons, New York (1992) p. 418-442.

[7] J. L. Amorós, A. Blasco, J. E. Navarro, V. Beltran, A. B. Escardino, Técnica Cerámica 105 (1982) 792-812.

[8] S. J. Lukasiewicz, J. S. Reed, Am. Ceram. Soc. Bull. 57, 9 (1978) 798-801.

[9] G. L. Messing, C. J. Markhoff, L. G. McCoy, Am. Ceram. Soc. Bull. 61 (1982) 857-860.

[10] R. L. K. Matsumoto, Engineered Materials Handbook Ceramics and Glass, ASM International, USA, 4 (1991) 128-129.

[11] G. P. Souza, R. Sanchez, J. N. F. Holanda, Cerâmica 48, 306 (2002) 102-107.

[12] ABNT, NBR 7181-84, Solo: Análise Granulométrica, (1984). [13] ABNT, NBR 7180-84, Solo: Massa Específica Real dos Grãos, (1984).

[14] JCPDS-ICCD, (1995).

[15] U. U. Gomes, Tese de Doutorado, UNICAMP-IFGW, Campinas, SP (1987).

[16] G. F. Bocchini, Powder Metallurgy 42, 2 (1999) 171-180.

[17] M. Radeka, J. Ramogajec, R. M. Neducin, B. Zivanovic, Ceram. Int. 21 (1995) 249-255.

[18] F. Sandrolini, G. Moriconi, J. Mater. Sci. 14 (1979) 2811-2817. (Rec. 02/09/03, Ac. 10/10/03) 